\title{
DRAG REDUCTION AND HEATING
}

\author{
J.M. Floryan, S. Shadman, M.Z. Hossain \\ Department of Mechanical and Materials Engineering \\ Western University \\ London, Ontario, N6A5B9, Canada
}

\begin{abstract}
An external force is required to maintain the relative movement of horizontal plates. It is shown that this force is reduced when the plates are subject to a spatially distributed heating. The largest reduction occurs for heating wavelengths of the order of distance between the plates with its magnitude increasing proportionally to the second power of the relevant Rayleigh number. It is shown that a sufficiently strong heating eliminates the need for the driving force altogether. The effect is active only in small Reynolds number flows.
\end{abstract}

\section{Keywords - drag reduction; heating}

\section{INTRODUCTION}

Friction between moving shafts found in numerous machines contributes to the energy cost of operating these devices. This cost may be estimated by determining force required to maintain the relative motion between these parts. Similar processes can be found in other applications, e.g. the towing of a free-floating body in a shallow basin. In general, flows which form in the space between two infinite plates in relative motion are well approximated by the Couette flow model, which is one of the family of simple flows frequently used in analyses.

Couette flow is characterized by the absence of a streamwise pressure gradient, a linear velocity distribution across the fluid layer and the lack of the linear stability limit [1]. The nonlinear stability analyses are well reviewed in [2] and demonstrate various routes to secondary finite-amplitude states as well as to turbulence. Surface modifications either in the form of transverse grooves [3], longitudinal grooves [4] or wall transpiration [5] can lead to centrifugal instabilities. Replacing the plane Couette flow with the annular Couette flow leads to shear instabilities [6]. Transition to secondary states leads to an increase in the wall shear and the need to increase the externally-imposed driving force. Such states should be avoided if minimization of energy cost is of interest.

Analyses of non-isothermal Couette flows are rather limited. They typically involve fluids with temperaturedependent material properties in a flow system exposed to a spatially uniform heating [7]. The addition of gravity brings in buoyancy effects which may generate secondary flows through the Rayleigh-Bénard (RB) instability [8,9] if the relevant critical conditions are met. Analyses of the resulting mixed convection are well reviewed in [10]. The use of spatially nonuniform heating leads to structured convection which occurs regardless of the heating intensity, but which is yet to be studied in the case of Couette flow. It is known that such heating leads to the reduction of pressure losses in pressuregradient driven flows [11-13].

The present work is part of a wider search for drag reducing methods which, in the present case, manifests itself in the form of reduction of the external force required to maintain the relative plate movement. One of the approaches is to assure stability of the flow so that transition to secondary states is avoided. Another approach, which is followed here, is to create spatial flow modulations which could lead to the reduction of shear and, thus, reduction of the driving force. The use of grooves for this purpose has been explored in [14-16]. This paper explores modulations created by spatially distributed heating.

\section{PROBLEM FORMULATION}

Consider two horizontal plates moving relative to each other and separated by a distance $2 h^{*}$ as shown in Fig. 1 .

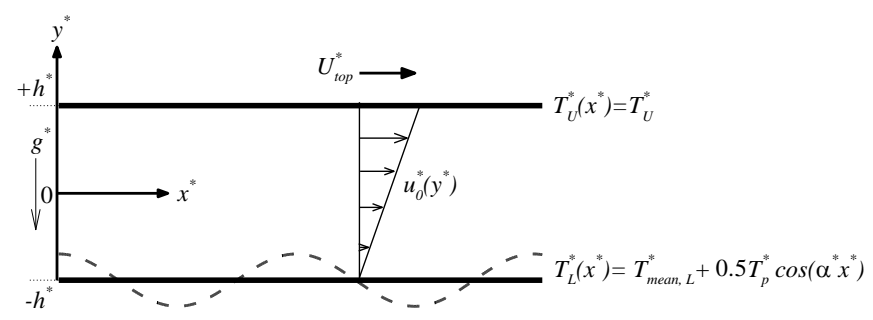

Figure 1. Schematic diagram of the flow domain.

The resulting gap extends to $₫ \infty$ in the $x$-direction and is filled with a fluid of thermal conductivity $k^{*}$, specific heat $c^{*}$, thermal diffusivity $\kappa^{*}=k^{*} / \rho^{*} c^{*}$, kinematic viscosity $v^{*}$, dynamic viscosity $\mu^{*}$, thermal expansion coefficient $\Gamma^{*}$ and with variations of density $\rho^{*}$ described by the Boussinesq approximation. The gravitational acceleration $g$ acts in the negative $y$-direction. The upper plate is pulled in the positive $\mathrm{x}$ direction with a constant velocity $U_{\text {top }}^{*}$ while the lower plate is stationary. When the system is isothermal, the drag force and the resulting flow field can be easily determined: 
$v_{0}(x, y)=\left[u_{0}(y), 0\right]=\left[\frac{(1+y)}{2}, 0\right], p_{0}(x, y)=C, F_{0}=0.5(1)$

where velocity has been scaled with $U_{t o p}^{*}$ as the velocity scale, $Q_{0}$ stands for the flow rate scaled with the same velocity scale, $\tau_{0}$ stands for the shear acting on the upper plate scaled with $U_{\text {top }}^{*} \mu^{*} / h^{*}, F_{0}$ denotes the force per unit length and width required to drag the upper plate scaled with $U_{\text {too }}^{*} \mu^{*} / h^{*}$ and the relevant Reynolds number is defined as $R e=U_{t o p}^{*} h / v$.

Introduce an external heating resulting in sinusoidal temperature variations along the lower plate,

$$
T_{L}^{*}\left(x^{*}\right)=T_{\text {mean }, L}^{*}+0.5 T_{p}^{*} \cos \left(\alpha^{*} x^{*}\right), T_{U}^{*}\left(x^{*}\right)=T_{U}^{*},
$$

where the subscripts "mean" and " $p$ " refer to the mean and periodic parts, respectively, $T_{p}^{*}$ is the peak-to-peak amplitude of the periodic component, and subscripts $L$ and $U$ refer to the lower and upper plates, respectively. Using the upper plate's temperature for reference and introducing the relative temperature $\theta * T^{*}-T_{U}^{*}$ lead to plates' temperatures of the form

$\theta_{L}^{*}(x)=\theta_{\text {uni }}^{*}+0.5 \theta_{p}^{*} \cos \left(\alpha^{*} x^{*}\right), \quad \theta_{U}^{*}(x)=0$,

where $\theta_{\text {uni }}^{*}=T_{\text {mean } L}^{*}-T_{U}^{*}, \theta_{p}^{*}=T_{p}^{*}$. Using half of the gap height $h^{*}$ as the length scale and $\kappa^{*} v^{*} /\left(g^{*} \Gamma^{*} h^{*}\right)$ as the temperature scale results in the temperature boundary conditions of the form

$$
\theta_{L}(x)=R a_{\text {uni }}+0.5 R a_{p} \cos (\alpha x), \quad \theta_{U}(x)=0,
$$

where $R a_{\text {uni }}=g^{*} \Gamma^{*} h^{*} T_{\text {uni }}^{*} /\left(\kappa^{*} v^{*}\right)$ is the uniform Rayleigh number measuring the intensity of the uniform heating, $R a_{p}=g^{*} \Gamma^{*} h^{*} T_{p}^{*} /\left(\kappa^{*} v^{*}\right)$ is the periodic Rayleigh number measuring the intensity of the periodic heating, and all material properties are evaluated at the reference temperature $T_{U}^{*}$.

The field equations take the form

$\frac{\partial u}{\partial x}+\frac{\partial v}{\partial y}=0, \quad u \frac{\partial u}{\partial x}+v \frac{\partial u}{\partial y}=-\frac{\partial p}{\partial x}+\nabla^{2} u$,

$u \frac{\partial v}{\partial x}+v \frac{\partial V}{\partial y}=-\frac{\partial p}{\partial y}+\nabla^{2} v+P^{-1} \theta, u \frac{\partial \theta}{\partial x}+v \frac{\partial \theta}{\partial y}=P^{-1} \nabla^{2} \theta$,

where $(u, v)$ are the velocity components in the $(x, y)$ directions, respectively, scaled with $U_{v}^{*}=v^{*} / h^{*}$ as the velocity scale, $p$ stands for the pressure scaled with $\rho^{*} U_{v}^{* 2}$ as the pressure scale and $\operatorname{Pr}=v^{*} / \kappa^{*}$ is the Prandtl number. These equations are subject to boundary conditions (2.4) combined with

$u(-1)=0, u(1)=R e, v(-1)=0, v(1)=0$,

where $U_{\text {top }}^{*} / U_{W}^{*}=R e$. As the flow is driven by the movement of the upper plate, any effects associated with the mean pressure gradient are eliminated through imposition of constraint of the form

$$
\left.\frac{\partial p}{\partial x}\right|_{\operatorname{men}}=0 \text {. }
$$

Heating alters the flow resulting in the change of the driving force, $\Delta F^{*}=F_{0}^{*}-F^{*}$, which, when scaled with $\rho^{*} U_{v}^{* 2}$, can be evaluated using the following relation

$$
\Delta F=F_{0}-F=\operatorname{Re}\left(\frac{1}{2}-\left.\operatorname{Re}^{-1} \lambda^{-1} \int_{0}^{\lambda} \frac{d u}{d y}\right|_{y=1} d x\right) .
$$

\section{DISCUSSION OF RESULTS}

Identical mean temperatures of both plates correspond to $R a_{u n i}=0$. When the upper plate is stationary $(R e=0)$, a purely periodic heating results in the formation of convective counterrotating rolls with the fluid moving upwards above the hot spots and downwards above the cold spots, as illustrated in Fig.2a, and its temperature rising above the mean in most of the fluid volume. Slow movement of the upper plate $(R e=1)$ results in a competition between the plate-driven and the buoyancy-driven motions. The flow topology is simple in the zones with the clockwise-rotating rolls as the roll movement is kinematically consistent with the plate movement, resulting in the formation of a single stream of fluid moving in the positive $x$-direction located in the immediate vicinity of the moving plate. A complex flow topology forms in the zones with the counterclockwise-rotating rolls as the fluid stream splits into two branches, one flowing above the rolls and one flowing beneath them. The upper branch is dominated by the plate effect, and the lower branch is dominated by the roll effect (see Fig.2b). Most of the fluid remains trapped in the rolls, i.e. either in the clockwise rolls attached to the lower plate or in the counter-clockwise rolls bounded by the two branches of the stream moving to the right. A further increase of the plate velocity $(R e=5)$ results in the dominance of the plate-driven movement with most of the fluid moving to the right, the elimination of the counterclockwise rolls and the reduction of the size of the clockwise rolls (see Fig.2c) but with the buoyancy effects still providing a significant contribution to the overall flow dynamics. A further increase of $R e$ results in the eventual elimination of the rolls (see topology for $R e=50$ in Fig.2D). The sequence of plots displayed in Fig.2 illustrates the process of formation of both the flow and(4hearkall boundary layers near the lower plate as $R e$ increases.

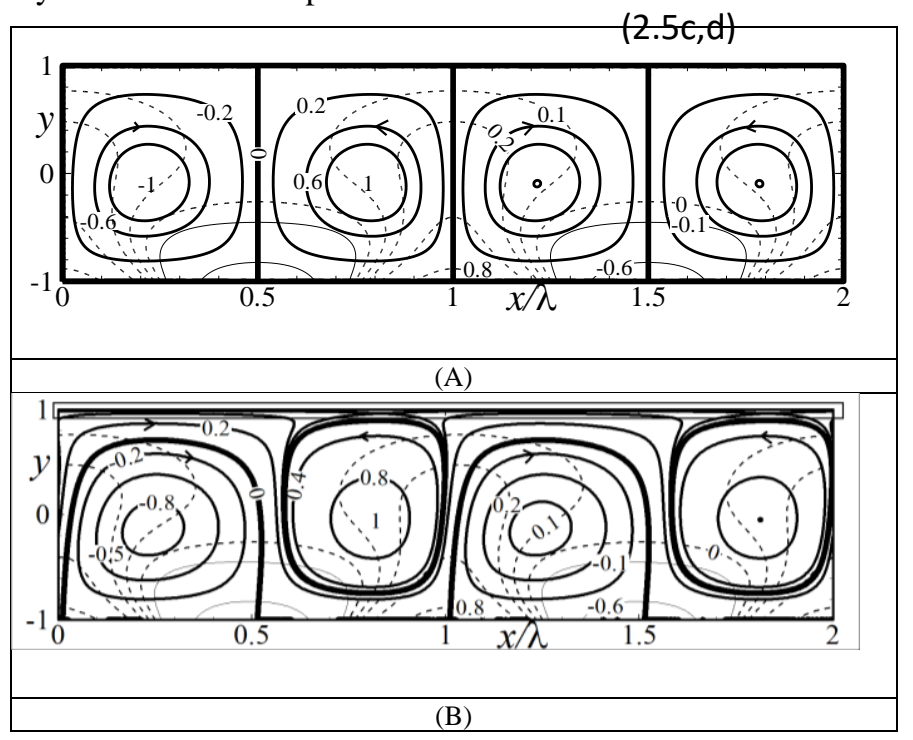




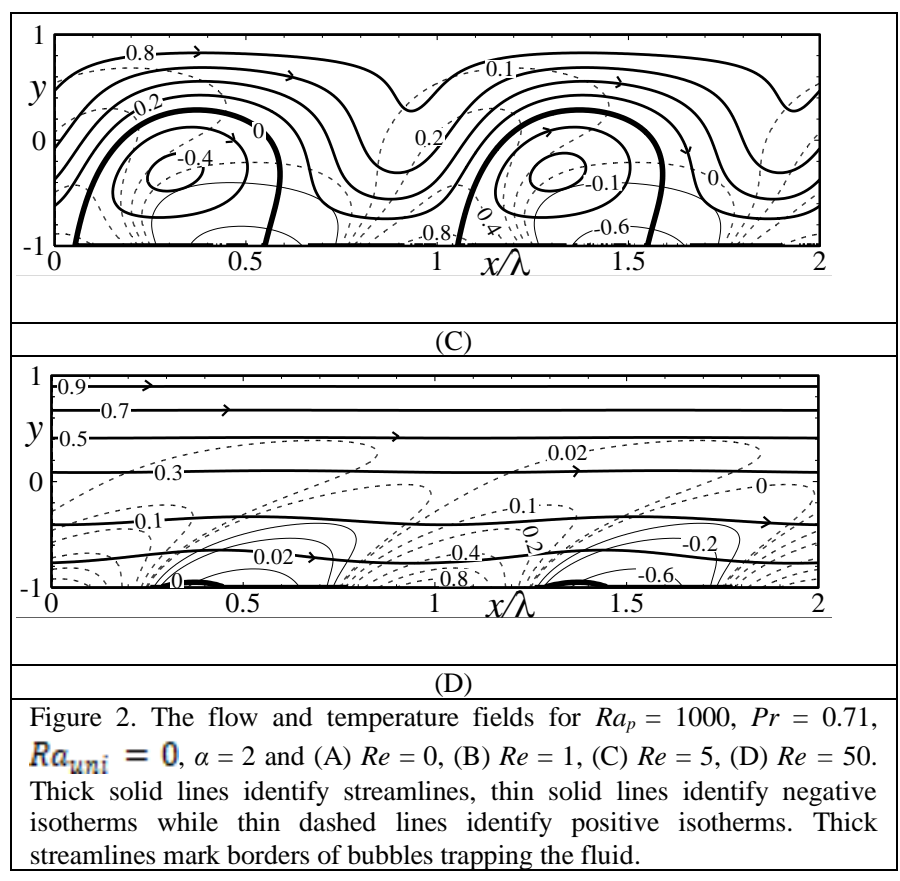

The results presented in Fig.3 demonstrate that all heating wave numbers lead to a decrease of the driving force with the magnitude of this reduction being a strong function of $\alpha$. The largest reduction occurs for $\alpha \approx 1-2$ with its magnitude decreasing proportionally to $\alpha^{4}$ if an excessively small $\alpha$ is used. The flow and temperature fields (not shown) are qualitatively similar to those displayed in Fig. 2. The use of an excessively large $\alpha$ also results in a reduction of $\Delta F$ but at a much higher rate, i.e. proportionally to $\alpha^{-7}$.

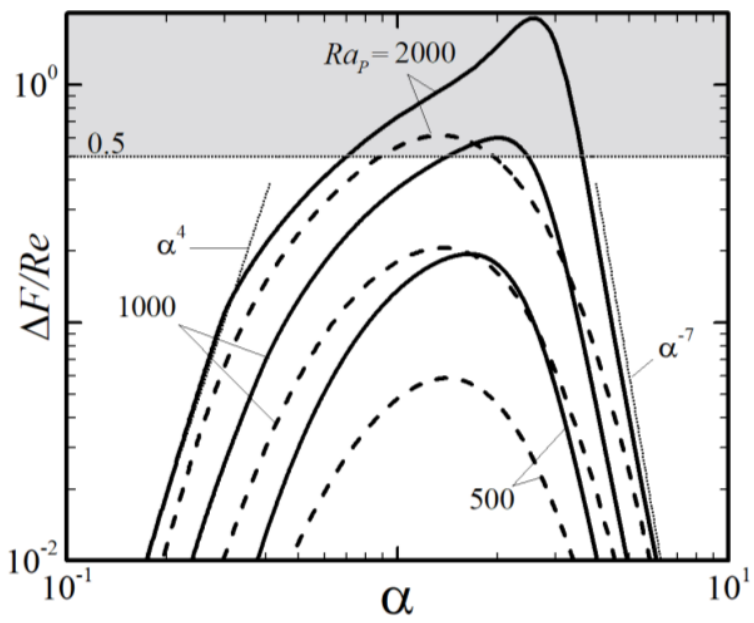

Figure 3. Variations of $\Delta F / R e$ as a function of $\alpha$ for $P r=0.71, R a_{b n f}=0, R e$ $=1$ (solid lines) and $R e=10$ (dashed lines). Thin dotted lines identify asymptotes. The shaded area identifies conditions where the driving force must change direction and becomes a braking force.

The force-reducing effect is a strong function of $R e$ (see Fig.4). The magnitude of $\Delta F$ increases proportionally to $R e$ for small $R e$ 's, reaches a maximum at $R e \sim 5-6$ and then decreases at a rate proportional to $R e^{-2}$. The flow topologies displayed in Fig. 2 show that the elimination of $\Delta F$ is associated with the reduction of convection bubbles and confinement of convection effects to a thin boundary layer near the lower plate.

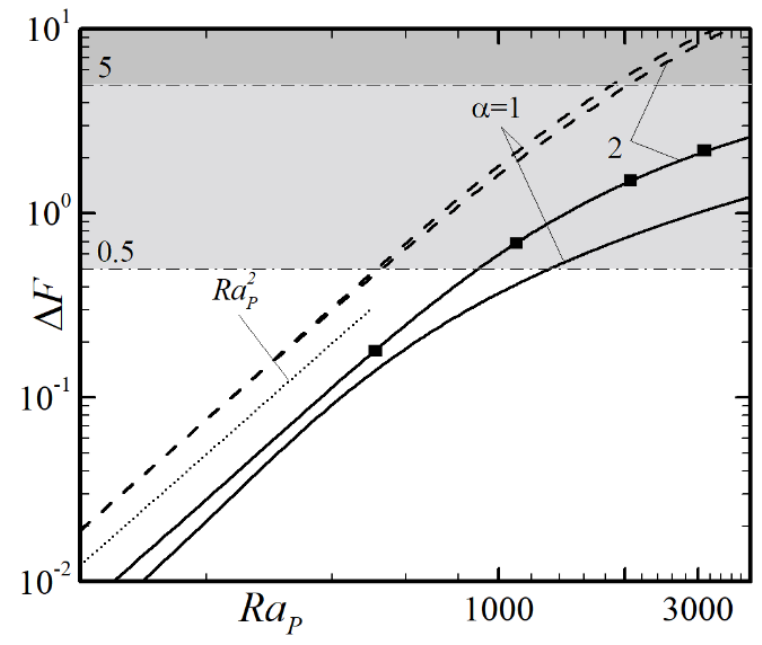

Figure 4. Variations of $\Delta F$ as a function of $R a_{p}$ for $P r=0.71, R a_{\text {bint }}=0, R e=$ 1 (solid lines) and $R e=10$ (dashed lines). The shaded area identifies conditions where the driving force must change direction and becomes a braking force when $R e=1$ and the double shaded area identifies such conditions for $R e=10$.

As it is unlikely that the mean temperatures of both plates can be kept equal, it is of interest to determine how their difference may affect the system response. This difference is expressed in the analysis as the uniform Rayleigh number $R a_{\text {wni }}$ whose positive (negative) values correspond to the lower plate being hotter (cooler). The results displayed in Fig.5 demonstrate that the uniform heating increases $\Delta F$ while cooling decreases it, and the change is approximately linear with $R a_{\text {uni }}$.

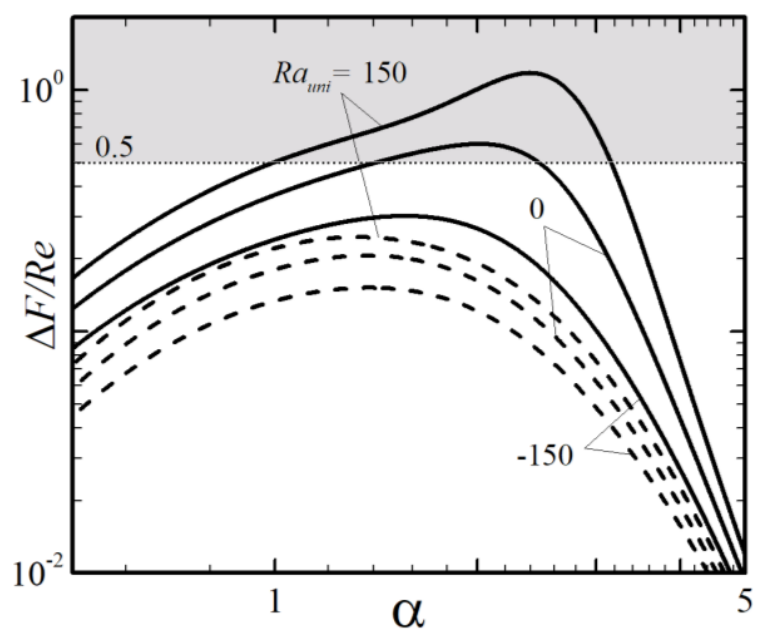

Figure 5. Variations of $\Delta F / R e$ as a function of $\alpha$ for $R e=1$ (solid lines) and $R e$ $=10$ (dashed lines), $R a_{P}=1000, \operatorname{Pr}=0.71$. The shaded area identifies conditions where the driving force changes direction and becomes a braking force. 


\section{SUMMARY}

An external force is required to generate a relative motion between horizontal plates with the rate of this motion expressed in terms of the Reynolds number $R e$. The effects of spatially distributed heating on the magnitude of this force have been analyzed. Heating resulting in the lower plate temperature varying sinusoidally in the horizontal direction, with its amplitude expressed in terms of a periodic Rayleigh number $R a_{p}$ and the spatial distribution described by the wave number $\alpha$, has been considered. The analysis has been limited to $R a_{p}<$ 3000 to avoid condition leading to a potential formation of secondary flows. The difference between the mean plates' temperatures has been expressed in terms of the uniform Rayleigh number $R a_{\text {uni }}$ with positive values corresponding to a warmer lower plate. The fluid motion results from a competition between the buoyancy-driven effects and the platedriven movement. The former has the form of counter-rotating rolls whose distribution is dictated by the heating pattern. The latter one adds a rectilinear motion which leads to the reduction and eventual elimination of the rolls if $R e$ is large enough.

It has been shown that periodic heating always reduces the driving force, regardless of whether the heating is applied to the lower or upper plate, but the magnitude of this reduction is a strong function of the heating wave number. The largest reduction is achieved for $\alpha=1-2$ with a rapid decrease of this effect when either too small or too large $\alpha$ 's are used. An increase in $R a_{\text {wni }}$ magnifies this effect. An increase in $R e$ eliminates the rolls and reduces this effect, leading to its practical elimination for $R e>30-50$ depending on the heating intensity. The use of proper heating intensity and distribution results in the complete elimination of the driving force as the plate movement can be supported by the buoyancy effects only. Conditions where an external braking force needs to be used to prevent the plate from accelerating have also been identified.

\section{ACKNOWLEDGMENT}

This work has been carried out with the support of NSERC of Canada.

\section{REFERENCES}

[1] [1] V.A. Romanow, Stability of Plane-Parallel Couette Flow, Funct. Anal. Applics. 7, 137-146 (1972).

[2] K. Deguchi, M. Nagata, Bifurcations and Instabilities in Sliding Couette flow, J. Fluid Mech. 678, 156-178 (2011).

[3] J.M. Floryan, Centrifugal Instability of Couette Flow over a Wavy Wall, Phys. Fluids 14, 312-322 (2002).

[4] H.V. Moradi, J.M. Floryan, Sliding Couette Flow in a Ribbed Annulus, Phys. Fluids 28, DOI: 10.1063/ 1.4955101 (2016).

[5] J.M. Floryan, Wall-Transpiration-Induced Instabilities in Plane Couette Flow, J. Fluid Mech. 488, 151-188 (2003).

[6] P. Gittler, Stability of Poiseuille-Couette flow between Concentric Cylinders, Acta Mech. 101, 1-13 (1993).

[7] A.W. Ogunsola, B.A. Peter, Analytical Solution of Non-Isothermal Couette Flow between two Plates, IOSR Journal of Mathematics 10, 4448, (IOSR-JM) e-ISSN: 2278-5728, ISSN:2319-765X (2014).

[8] H. Bénard, Les Tourbillons Cellulaires dans une Nappe Liquide, Revue Générale Science Pure et Applique 11, 1261-1271 (1900).

[9] J.W.S. Rayleigh, J.W.S. On Convection Currents in a Horizontal Layer of Fluid, when the Higher Temperature is on the Under Side, Phil. Mag. 32, 529-546 (1916).

[10] R.E. Kelly, R.E. The Onset and Development of Thermal Convection in Fully Developed Shear Flows, Adv. App. Mech. 31, 35-112 (1994).

[11] M.Z. Hossain, D. Floryan, J.M. Floryan, Drag Reduction due to Spatial Thermal Modulations, J. Fluid Mech. 713, 398-419 (2012).

[12] D. Floryan, J.M. Floryan, Drag Reduction in Heated Channels, J. Fluid Mech. 765, 353-395 (2015).

[13] M.Z. Hossain, J.M. Floryan, Drag Reduction in a Thermally Modulated Channel, J. Fluid Mech. 791,122-153 (2016).

[14] M.J. Walsh, Drag Characteristics of V-groove and Transverse Curvature Riblets. In Viscous Drag Reduction (ed. G. R. Hough), AIAA J. 72, 168-184 (1980).

[15] M.J. Walsh, Riblets as a Viscous Drag Reduction Technique, AIAA J. 21, 485-486 (1983).

[16] A. Mohammadi, J.M. Floryan, J.M. Effects of Longitudinal Grooves on the Couette-Poiseuille flow, J. Theor. Comp. Fluid Mech. 28, 549-572 (2014). 